\title{
Photometric study of 61 totally eclipsing contact binaries from the ASAS, OGLE, HATNet, AST3 and TESS databases
}

\author{
J. Rukmini, D. Shanti Priya, M. Raghu Prasad and P. Ravi Raja \\ Department of Astronomy, Osmania University, Hyderabad-500007, India.
}

Received: October 28, 2019; Accepted: January 23, 2020

\begin{abstract}
Contact binaries form an interesting class of binaries which not only show mutual interactions through gravitationally bound periodic close orbits, but also dynamical interactions through mass transfer, angular momentum loss and modulation of their orbits due to the presence of tertiary components and magnetic activity. They are important as distance indicators and laboratories to study stellar evolutionary models. The current work highlights our photometric study of 61 totally eclipsing contact binaries from the ASAS, OGLE, HATNet, AST3 and TESS databases. Physical parameters are derived using PHOEBE. The selected binaries fall in a range of short periods $(0.34-0.97 \mathrm{~d})$, low mass ratios (0.076-0.504), F5-M0 spectral types, and a wide range of fillout factors (3-85\%). Based on obtained fillout factors, 5 were classified as shallow/marginal contact, 41 as over-contact and 15 as deep-contact binaries. The absolute parameters are compared with those of well-studied binaries. Possible evolutionary states are discussed.
\end{abstract}

Key words: binaries: contact - eclipses: total - O'Connell effect

\section{Introduction}

Eclipsing binaries in pre-contact and marginal contact phases are important systems to help understand close binary evolution from detached to contact stages. The contact binary systems with mass ratios $<0.25$ and fillout factors $>50 \%$ are prominent sources of interest, as they are progenitors of some fascinating objects and related to several key astrophysical processes (Liao et al., 2017).

\section{Data collection and analysis}

Photometric data of contact binaries with totality were collected from various archival databases like the All Sky Automated Sky Survey-3 (ASAS, Pojmanski, 2004), the Optical Gravitational Lensing Experiment-3 (OGLE, Udalski et al., 2008), the Hungarian Automated Telescope Network (HATNet, Hartman et al., 2004), the Antarctic Survey Telescope at Dome A (AST3, Wang et al., 2017), and the Transiting Exoplanet Survey Satellite (TESS,Stassun et al., 2018). We 
identified for further analysis a total of 61 variables from these databases (parameter ranges in Table 1) showing totality at one or both eclipses. Period

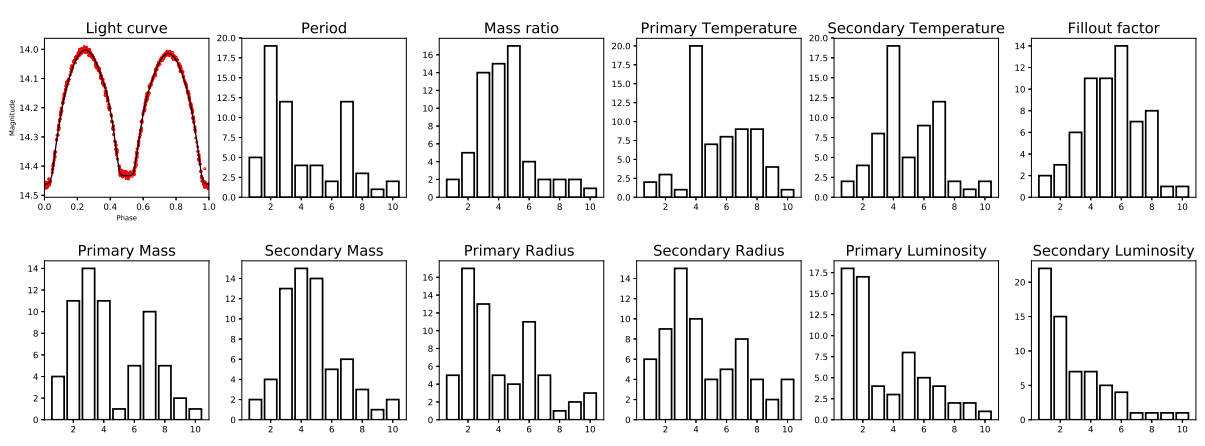

Figure 1. (a) Model light curve with observations shown as circles and the therotical fit as a solid line; (b) Histograms showing number distributions of various parametes for the 61 objects under study.

searches were performed using Persea (Schwarzenberg-Czerny, 1996) to derive ephemerides for all the variables. Phased light curves were then analysed using the software package PHysics Of Eclipsing BinariEs (PHOEBE) (Prša, 2006). Effective surface temperatures of the primary components $\left(T_{1}\right)$ were taken from Gaia2 (Brown et al., 2018) for the OGLE, HATNet and AST variables, calculated using Allen's tables (Allen \& Cox, 2000) for ASAS variables, and adopted from the TESS database (Stassun et al., 2018) for TESS variables. The gravity darkening coefficients $g_{1,2}$ (Lucy, 1967) and albedos $A_{1,2}$ (Rucinski, 1969) were adopted in accordance with the temperature of the stars. Adjusted parameters were $q, T_{2}, L_{1}, i$ and $\Omega$ and iterations were performed to get synthetic light curves with minimum $\Sigma(O-C)^{2}$ between observations and synthetic curves. For totally eclipsing binaries, Pribulla et al. (2003a) found that mass ratios derived from photometric data are comparable with those derived from spectroscopic analyses. Also, Terrell \& Wilson (2005) came to a similar conclusion for over-contact systems. Hence, for our systems, mass ratios determined from light curves can be considered to be reliable. During the analysis, 16 variables showing asymmetry in their light curves, i.e. an O'Connell effect, (O'Connell, 1951) were fitted with spots. Fillout factors were calculated using the equation $F=\left(\Omega_{\text {in }}-\Omega\right) /\left(\Omega_{\text {in }}-\Omega_{\text {out }}\right)$, where $\Omega_{\text {in }}$ and $\Omega_{\text {out }}$ are the potentials of the inner and outer Lagrangian equipotential surfaces, respectively. The variables were classified on the basis of fillout factors as shallow/marginal, over-contact and deep-contact binaries. The absolute parameters were derived using the Gazeas (2009) relations. 
Table 1. Range of Parameter Values

\begin{tabular}{llll}
\hline \hline & ASAS & OGLE & HATNet \\
\hline No. of Objects & 2 & 48 & 3 \\
Field & disk & bulge & disk \\
$P$ (days) & $0.3787-0.7419$ & $0.3486-0.9598$ & $0.4258-0.9731$ \\
Distance $(\mathrm{pc})$ & $227.8-321.1$ & $659.2-3003.9$ & $891.4-11299.4$ \\
$T_{1}(\mathrm{~K})$ & $5475-6475$ & $4092-6512$ & $5574-6541$ \\
$q$ & $0.197-0.220$ & $0.132-0.380$ & $0.096-0.180$ \\
$\Delta T=T_{1}-T_{2}(\mathrm{~K})$ & $68-316$ & $48-812$ & $282-1009$ \\
$F(\%)$ & $11-35$ & $3-85$ & $21-49$ \\
$M_{1}\left(M_{\odot}\right)$ & $1.29-2.11$ & $1.16-2.52$ & $1.42-2.65$ \\
$M_{2}\left(M_{\odot}\right)$ & $0.28-0.42$ & $0.21-0.64$ & $0.14-0.36$ \\
\hline \hline & AST3 & TESS & All \\
\hline No. of Objects & 6 & 2 & 61 \\
Field & disk & disk & bulge/disk \\
$P($ days $)$ & $0.3672-0.7808$ & $0.50449-0.53480$ & $0.3486-0.9731$ \\
Distance (pc) & $1536.8-4280.8$ & $738.2-1196.5$ & $227.8-11299.4$ \\
$T_{1}(\mathrm{~K})$ & $4897-5873$ & $5959-6450$ & $4092-6541$ \\
$q$ & $0.143-0.504$ & $0.076-0.129$ & $0.076-0.504$ \\
$\Delta T=T_{1}-T_{2}(\mathrm{~K})$ & $220-410$ & $415-508$ & $48-1009$ \\
$F(\%)$ & $18-31$ & $3-26$ & $3-85$ \\
$M_{1}\left(M_{\odot}\right)$ & $1.18-2.20$ & $1.71-1.72$ & $1.16-2.65$ \\
$M_{2}\left(M_{\odot}\right)$ & $0.26-0.59$ & $0.13-0.22$ & $0.13-0.64$ \\
\hline & & & \\
\hline
\end{tabular}

\section{Results and conclusions}

Light curve analyses were perfomed on 61 short period $(P<1 \mathrm{~d})$, totally eclipsing contact binaries of later spectral types (F5-M0) using PHOEBE. The distribution of basic parameters is shown in Fig. 1 and listed in Table 1. We found that 16 variables were showing light curve asymmetries near maxima (O'Connell effect), and best fit solutions were obtained after incorporating starspots. Furthermore, the derived absolute parameters show that primary components are more massive than their companions. As all the binaries in this study are totally eclipsing, the derived mass ratios and basic parameters are more reliable. Most of the binaries in this study are found to be low mass ratio systems with primaries the more massive of the two stars. One system in particular shows a very low mass ratio $(q \sim 0.07)$ and is similar to some of the well-known low mass ratio systems (Oh et al., 2007). Many of our systems are interesting sources for follow-up observations and for improving binary evolutionary models.

From the solutions we found 15 deep-contact $(F>50 \%), 41$ over-contact $(50 \%>F>10 \%)$ and 5 shallow/marginal contact binaries $(\mathrm{F}<10 \%)$. The ob- 

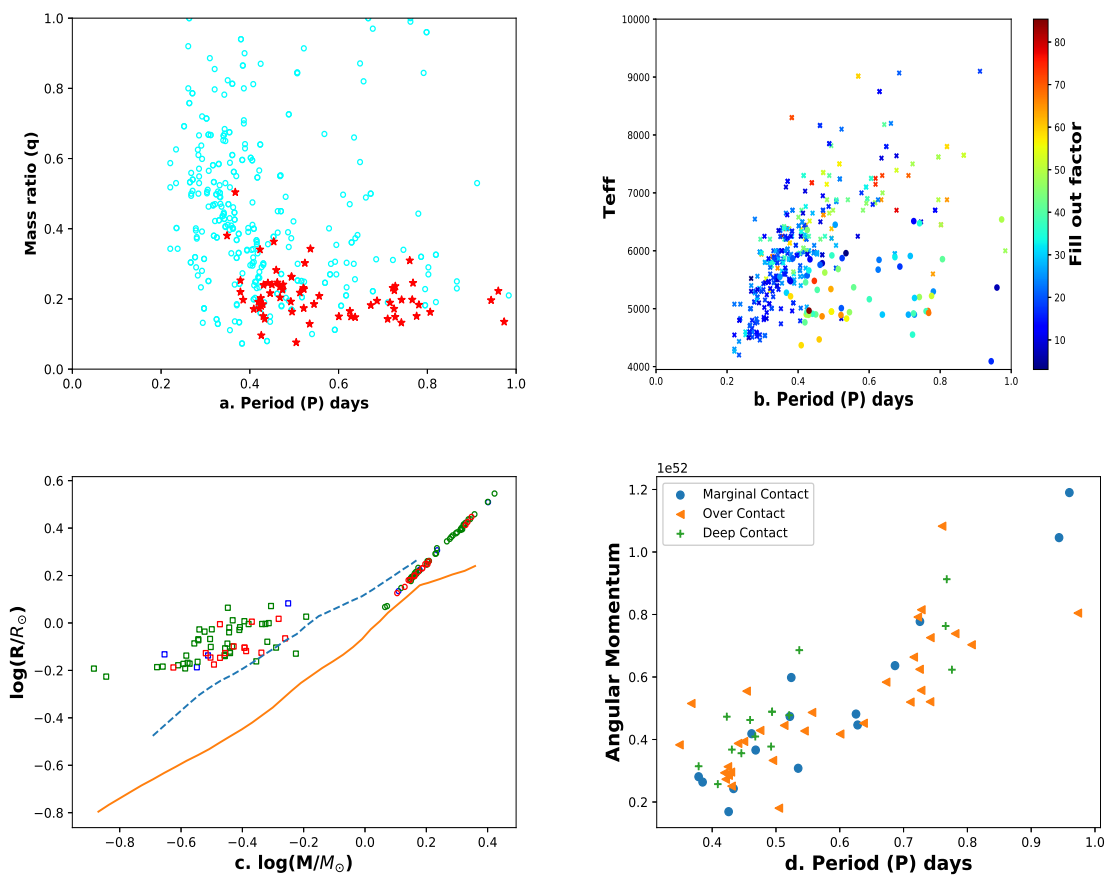

Figure 2. a. Orbital period vs. mass ratio for contact binaries, with red symbols indicating the 61 objects of this study, and circles representing the database of well studied contact binaries (Pribulla et al., 2003b; Csizmadia \& Klagyivik, 2004; Deb \& Singh, 2011); b. Orbital period vs. effective temperature, with colored bars representing fillout factors; c. $\log M$ vs. $\log R$, with circles for primary and squares for secondary components, and blue indicating marginal contact, green over-contact, and red deep-contact binaries. Solid and dotted lines represent ZAMS and TAMS stars; d. Orbital period vs. total angular momentum of the binaries.

jects in the direction of the Galactic bulge were all over-contact systems. Large temperature differences between components are observed in a few systems with low fillout factors and could be due to their evolutionary state of transition between contact and broken contact phases, as explained by the thermal relaxation oscillation (TRO) model. The $P$ vs. $q$ (Fig. 2) plot shows that most of the binaries are A-type W UMa systems, however with a wide range of fillout factors. The $\log M$ vs. $\log R$ and $P$ vs. $T_{\text {eff }}$ (Fig. 2) plots show that most of the systems are evolved and have secondaries that are overluminous for their main sequence masses. The results add to the existing evidence that in short period contact binaries, late-type systems are more frequent than early type systems. Studying a larger number of objects in the short period cut-off range will allow correlations to be tested and help the development of better evolutionary models. 
Acknowledgements. We acknowledge the archival databases of ASAS, OGLE, HATNet, AST3D, TESS and Gaia2 and the financial support provided for this research by DST (Govt. of India) SERB grant EEQ/2017/000411.

\section{References}

Allen, C. W. \& Cox, A. N. 2000, Allen's astrophysical quantities (Springer Science \& Business Media)

Brown, A., Vallenari, A., Prusti, T., et al., Gaia Data Release 2-Summary of the contents and survey properties. 2018, Astronomy $\&$ astrophysics, 616, A1

Csizmadia, S. \& Klagyivik, P., On the properties of contact binary stars. 2004, Astronomy \& Astrophysics, 426, 1001

Deb, S. \& Singh, H. P., Physical parameters of 62 eclipsing binary stars using the All Sky Automated Survey-3 data- I. 2011, Monthly Notices of the Royal Astronomical Society, 412, 1787

Gazeas, K., Physical parameters of contact binaries through 2-D and 3-D correlation diagrams. 2009, Communications in Asteroseismology, 159, 129

Hartman, J., Bakos, G., Stanek, K., \& Noyes, R., HATNET Variability Survey in the High Stellar Density Kepler Field with Millimagnitude Image Subtraction Photometry. 2004, The Astronomical Journal, 128, 1761

Liao, W.-P., Qian, S.-B., Soonthornthum, B., et al., TYC 1337-1137-1 and TYC 3836-0854-1: Two Low-mass Ratio, Deep Overcontact Systems Near the End Evolutionary Stage of Contact Binaries. 2017, Publications of the Astronomical Society of the Pacific, 129, 124204

Lucy, L., Gravity-darkening for stars with convective envelopes. 1967, Zeitschrift fur Astrophysik, 65, 89

O'Connell, D., The so-called periastron effect in close eclipsing binaries; New variable stars (fifth list). 1951, Publications of the Riverview College Observatory, $\mathbf{2}, 85$

Oh, K.-D., Kim, C.-H., Kim, H.-I., \& Lee, W.-B., The Low-Mass-Ratio Contact Binaries-V410 Aur, V776 Cas, HN UMa, II UMa. in , The Seventh Pacific Rim Conference on Stellar Astrophysics, Vol. 362, 82

Pojmanski, G., The all sky automated survey. the catalog of variable stars. ii. $6 \mathrm{~h}-12 \mathrm{~h}$ quarter of the southern hemisphere. 2004, arXiv preprint astro$p h / 0401125$

Pribulla, T., Kreiner, J., \& Tremko, J., Catalogue of the field contact binary stars. 2003a, Contributions of the Astronomical Observatory Skalnate Pleso, 33, 38 
Pribulla, T., Kreiner, J., \& Tremko, J., VizieR Online Data Catalog: Catalogue of field contact binary stars (Pribulla+, 2003). 2003b, VizieR Online Data Catalog, $\mathbf{5 1 1 9}$

Prša, A., PHOEBE Scientific Reference. 2006, PHOEBE version 0.30, University of Ljubljana, Faculty of Mathematics and Physics, Dept. of Astrophysics

Rucinski, S., The proximity effects in close binary systems. II. The bolometric reflection effect for stars with deep convective envelopes. 1969, Acta Astronomica, 19, 245

Schwarzenberg-Czerny, A., Fast and statistically optimal period search in uneven sampled observations. 1996, The Astrophysical Journal Letters, 460, L107

Stassun, K. G., Oelkers, R. J., Pepper, J., et al., The TESS Input Catalog and Candidate Target List. 2018, The Astronomical Journal, 156, 102

Terrell, D. \& Wilson, R. E., Photometric Mass Ratios of Eclipsing Binary Stars. 2005, Astrophys. Space Sci., 296, 221, DOI: 10.1007/s10509-005-4449-4

Udalski, A., Szymanski, M., Soszynski, I., \& Poleski, R., The optical gravitational lensing experiment. final reductions of the OGLE-III Data. 2008, arXiv preprint arXiv:0807.3884

Wang, L., Ma, B., Li, G., et al., Variable Stars Observed in the Galactic Disk by AST3-1 from Dome A, Antarctica. 2017, The Astronomical Journal, 153, 104 